Spectroscopic Characterization and Simulation of Chemical Sputtering Using the DiMES Porous Plug Injector in DIII-D

A. G. McLean, J. W. Davis, P. C. Stangeby, N. H. Brooks, D. G. Whyte, S. L. Allen, B. D. Bray, S. Brezinsek, J. D. Elder, M. E. Fenstermacher, M. Groth, A. A. Haasz, E. M. Hollmann, R. Isler, C. J. Lasnier, D. L. Rudakov, J. G. Watkins, W. P. West, C. P. C. Wong

May 17, 2006

Journal of Nuclear Material 
This document was prepared as an account of work sponsored by an agency of the United States Government. Neither the United States Government nor the University of California nor any of their employees, makes any warranty, express or implied, or assumes any legal liability or responsibility for the accuracy, completeness, or usefulness of any information, apparatus, product, or process disclosed, or represents that its use would not infringe privately owned rights. Reference herein to any specific commercial product, process, or service by trade name, trademark, manufacturer, or otherwise, does not necessarily constitute or imply its endorsement, recommendation, or favoring by the United States Government or the University of California. The views and opinions of authors expressed herein do not necessarily state or reflect those of the United States Government or the University of California, and shall not be used for advertising or product endorsement purposes. 


\title{
Spectroscopic characterization and simulation of chemical sputtering using the DiMES Porous Plug Injector in DIII-D
}

\author{
A.G. McLean, ${ }^{a}$ J.W. Davis, ${ }^{a}$ P.C. Stangeby, ${ }^{a}$ N.H. Brooks, ${ }^{b}$ D.G. Whyte, ${ }^{c}$ \\ S.L. Allen, ${ }^{d}$ B.D. Bray, ${ }^{b}$ S. Brezinsek, ${ }^{e}$ J.D. Elder, ${ }^{a}$ M.E. Fenstermacher, ${ }^{d}$ \\ M. Groth, ${ }^{d}$ A.A. Haasz, ${ }^{a}$ E.M. Hollmann, ${ }^{f}$ R. Isler, ${ }^{g}$ C.J. Lasnier, ${ }^{d}$ \\ D.L. Rudakov, ${ }^{f}$, J.G. Watkins, ${ }^{h}$ W.P. West, ${ }^{b}$ C.P.C. Wong ${ }^{b}$ \\ ${ }^{a}$ University of Toronto Institute for Aerospace Studies, Toronto, M3H 5T6, Canada. \\ ${ }^{b}$ General Atomics , San Diego, California 92186-5608, USA. \\ ${ }^{c}$ University of Wisconsin, Madison, Wisconsin 53706, USA. \\ ${ }^{d}$ Lawrence Livermore National Laboratory , Livermore, California 94550, USA. \\ ${ }^{e}$ Institut fuer Plasmaphysik Forschungszentrum, Juelich GmbH 52425, Juelich,Germany. \\ ${ }^{f}$ University of California, San Diego, California 92093, USA. \\ ${ }^{g}$ Oak Ridge National Laboratory, Oak Ridge, Tennessee 37831, USA. \\ ${ }^{\text {h}}$ Sandia National Laboratory, Albuquerque, New Mexico 87185, USA.
}

\begin{abstract}
A self-contained gas injection system for the Divertor Material Evaluation System (DiMES) on DIII-D has been employed for in-situ study of chemical erosion in the tokamak divertor environment. The Porous Plug Injector (PPI) releases methane, a major component of molecular influx due to chemical sputtering of graphite, from the tile surface into the plasma at a controlled rate through a porous graphite surface. Perturbation to local plasma is minimized, while also simulating the immediate environment of methane molecules released from a solid graphite surface. The release rate was chosen to be of the same order of magnitude as natural sputtering.

Photon efficiencies of $\mathrm{CH}_{4}$ for measured local plasma conditions are reported. The contribution of chemical versus physical sputtering to the source of $\mathrm{C}^{+}$at the target is
\end{abstract}


assessed through measurement of $\mathrm{CII}$ and $\mathrm{CD} / \mathrm{CH}$ band emissions during release of $\mathrm{CH}_{4}$ from the PPI, and due to intrinsic emission.

JNM keywords: Carbon, Fusion reactor materials, Plasma Materials Interaction, Surface effects, Theory and Modeling

PSI-17 keywords: Carbon impurities, DIII-D, Hydrocarbons

PACS:

Corresponding author address: Adam McLean, 4925 Dufferin Street, Toronto, Ontario, Canada M3H 5 T6

Corresponding author e-mail: adam.mclean@utoronto.ca

Presenting author address: Adam McLean, 4925 Dufferin Street, Toronto, Ontario, Canada M3H 5 T6

Presenting author e-mail: adam.mclean@utoronto.ca

\section{Introduction}

Carbon plasma facing components (PFCs) in a tokamak are subject to chemical erosion due to sputtering from hydrogenic impact. Although many laboratory-based measurements of erosion yields have been carried out, their applicability to the tokamak environment is uncertain due to tokamak-specific mechanisms, such as prompt redeposition of dissociated hydrocarbon fragments. A number of methane injection experiments have been carried out on various tokamaks with gas injection using nozzles or tile gaps. These macroscopic openings can be larger than the mean-free-path for deposition of the molecular fragments resulting from the break-up of the hydrocarbon molecules due to interaction with plasma electrons and ions. In order to better reproduce the immediate environment experienced by a hydrocarbon molecule released by chemical 
sputtering from an essentially continuous carbon surface, a Porous Plug Injector (PPI) has been used to inject the hydrocarbon gas in these experiments. Such a spatially distributed injection of the gas should also be less disturbing to local plasma conditions than occurs with nozzle injection.

\section{Experiment}

The PPI is a self contained gas injection system capable of puffing minute quantities of gas into the lower divertor of DIII-D (Fig. 1). Motivation and design of the PPI are discussed in [1]. The PPI was operated in DIII-D for one dedicated run-day and several additional times in piggyback experiments. The dedicated day included exposure to 205 second duration Simple as Possible Plasma (SAPP) [2] L-mode repeat discharges, 15 with gas puffing from the PPI. The gas used in the PPI was a 80\% / 20\% mixture (by weight) of $\mathrm{CH}_{4}$ and He. The latter was employed for in-situ gas flow rate calibration through observation of the $6680 \AA$ HeI line whose S/XB-value is known for the expected local plasma conditions.

Each plasma shot included a 3-step sweep of the OSP, each with a minimum 0.5 second dwell for diagnostic characterization. Local plasma conditions at the OSP during PPI exposure were found to be well attached, with $T_{e}=22+/-5 \mathrm{eV}$ and $n_{e}=2.5+/-0.2 \times 10^{19} \mathrm{~m}^{-3}$ and $\Gamma_{D}^{+}=5 \times 10^{22} \mathrm{~m}^{-2} \mathrm{~s}^{-1}$. Expected carbon erosion yields at the OSP for these conditions are $Y_{D \rightarrow C}^{\text {phys }} \sim 0.015$ [3], and $Y_{D \rightarrow C}^{\text {chem }}<0.05$ [4]. The resulting total carbon atom influx is estimated to be $\Gamma_{c, i n} \sim 1 \times 10^{21} \mathrm{~m}^{-2} \mathrm{~s}^{-1}$, for a total release rate of $\sim 7 \times 10^{17} \mathrm{C} / \mathrm{s}$ over the surface area of the PPI, $7 \times 10^{-4} \mathrm{~m}^{2}$. At the design gas flow rate, the PPI will inject methane at a rate which equals this total carbon atom flux, $\sim 2$ standard cubic centimeters of $\mathrm{CH}_{4}$ per minute (sccm), or 0.025 torrL/s. 
The gas puff rate from the PPI was varied both from shot to shot and within each dwell period in order to determine the degree of perturbation that the puff has on local plasma conditions, and to identify trends in spectroscopic emission data.

The main DIII-D diagnostics used for the PPI experiment include a high resolution spectrometer (MDS), and the DiMES TV camera, both viewing the PPI almost directly from above. Langmuir probes, tangential cameras, filterscopes, divertor Thomson scattering, and an infrared camera were also used for plasma and plasma surface characterization.

\section{Observations}

The gas puff rate from the PPI was determined by a combination of ex-situ calibration of the driving voltage, in-situ calibration of gas canister pressure during controlled leak periods, and comparison of the derived flow rate to HeI emission during plasma operation. While the intention was to operate the PPI primarily with the (low) flow rates described above, difficulties were encountered in controlling such low flow rates in a thermally variable environment. The flow rate was maintained as originally targeted for a single shot, then was increased to $5-15 \mathrm{X}$ the targeted rate for 13 additional shots, and finally, was increased to 50X the targeted rate for a single shot. The major sources of uncertainty, including electrical noise and electrical pickup during ramps of nearby magnetic coils, resulted in an estimated error of $25 \%$ in the gas puff rates.

The MDS (spectral resolution $\Delta \lambda=0.1 \mathrm{~A}, 80 \AA$ bandwidth, $125 \mathrm{~ms}$ integration time) [5]

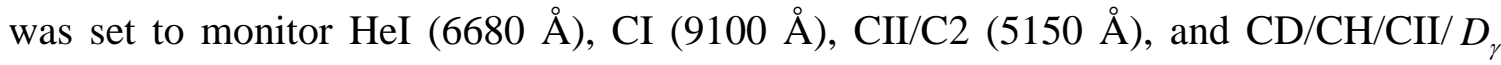
(4300 A) in successive plasma discharges. Simultaneous wide field (50 x $50 \mathrm{~cm}$ ) coverage was provided by the DiMES TV camera monitoring equivalent emission lines. 
Simultaneous observation by MDS was made of the PPI and of a radially equivalent location separated toroidally from DiMES by $25 \mathrm{~cm}$, allowing for an accurate background subtraction from emission data at the PPI. Observations and analysis of CI emission are discussed in [6]. A sample spectra from MDS viewing DiMES is shown in Fig. 2, demonstrating evolution from a CD-dominated Gëro band (CD bandhead at 4308 $\AA$ ), to $\mathrm{CH}$ (bandhead at $4313 \AA$ ) at the onset of the PPI puff.

While CD and CI emission were observed not to extend much beyond the circle of the PPI head on the divertor floor, CII emission from the puff was found to extend well beyond the spatial extent of the PPI, preferentially downstream. This spatial distribution, resulting from frictional drag with the background plasma, was accounted for by integration of emission over the background throughout the DiMES TV view, and applying a multiplication factor of $2.3(+/-0.3)$ to the integrated emissivity within the spatial bounds of the PPI alone.

After removing the PPI, its surface was found to be coated with an amorphous hydrocarbon layer of near uniform opacity, indicating that the OSP region had been altered from a zone of net erosion [7], to one of net deposition as a consequence of the gas puff. Spatial distribution of the deposit was clearly biased downstream, and slightly more intense on the radially outward side. Based on emission patterns seen by DiMES $\mathrm{TV}$, it is estimated that $\sim 90 \%$ of the injected carbon was locally deposited on the porous cap in a layer $\sim 350 \mathrm{~nm}$ thick [8].

Substantial $\mathrm{C}_{2}$ dimer emission due to the PPI puff was observed, despite the lack of $\mathrm{C}_{\mathrm{x}} \mathrm{H}_{\mathrm{y}}$, $x>1$ in the injected gas. This indicates the presence of significant surface interaction between injected $\mathrm{CH}_{4}$ molecules [9]. 
Initial examination of the $\mathrm{CD} / \mathrm{CH}$ spectra produced by the PPI puff found no significant increase in $\mathrm{CD}$ production as a consequence of $\mathrm{CH}_{4}$ injection and breakup, indicating that isotopic exchange between background $\mathrm{D}\left(\mathrm{D}^{+}\right.$, recycled $\mathrm{D}^{0}$, and $\mathrm{D}$ deposited in the surface) is rare [10].

\section{Discussion}

Simultaneous integration of the Gëro CD/CH $A \rightarrow X$ band (4270-4315 $\AA$ ) and CII (4267 $\AA$ ) line for both emission from the PPI gas injection and for intrinsic background sources provides a direct method for determination of relative contribution to the $\mathrm{C}^{+}$-source by chemical and physical sputtering, see Fig. 3. The measured relationship between integrated $\mathrm{CII}$ and $\mathrm{CD} / \mathrm{CH}$ intensity is found to be quite linear for all PPI puff rates, evidently an indication that the puff is not perturbative to the local plasma (Fig 3a). Because the PPI injects $\mathrm{CH}_{4}$ molecules only, a primary constituent of chemical sputtering, spectroscopic emission from the puff may be considered indicative of pure chemical erosion only. In Fig. 3b, the intrinsic CII and CD emission are shown indicating that half of the CII, i.e. C+-source, can be ascribed to chemical sputtering. As already noted, in the first application of the PPI, most of the puffing data in Fig 3a were obtained for higher injection rates than planned, and there is paucity of data in the low flow region needed to reach more definitive conclusions. This will be rectified in future experiments. In detached conditions it is clear, however, that the contribution of chemical sputtering is very small.

Further potential sources of error in this measurement include an accurate background subtraction over the broad $\mathrm{CD} / \mathrm{CH}$ spectra, full accounting of $\mathrm{CII}$ emission due to the puff 
which extends well beyond the PPI head, and a low photon detection threshold on MDS. The combined influence of these errors indicates that chemical sputtering contributes $\sim 30-90 \%$ of the $\mathrm{C}^{+}$-source in attached conditions and $\sim 5-15 \%$ for detached ones. In the next application of the PPI it is anticipated that it will be possible to significantly reduce these uncertainties.

Contribution to the $\mathrm{CD} / \mathrm{CH}$ spectrum by $\mathrm{D}_{2}$ as identified by Brezinsek [11] is thought to be due to intrinsic sources only [12] and was found to be a minor constituent compared to experimental error in measurement of the integrated $\mathrm{CD} / \mathrm{CH}$ emission.

It may be noted that the relative contributions of physical and chemical sputtering to the C-atom source entering the plasma has to be assessed separately. This will require carefully matched measurements of the CD and CI emission, which was not attempted in the present study, but will be one of the aims of future experiments.

Knowledge of photon flux specific to the local plasma conditions, $\phi\left(n_{e}, T_{e}\right)$, for each fragmented species monitored by MDS allows determination of molecular photon efficiencies, $\mathrm{D} / \mathrm{XB}$, for fragment species due to injection of $\mathrm{CH}_{4}$ at the DIII-D attached OSP. For attached plasma conditions investigated here, these are listed with estimated error based on the uncertainties mentioned above:

$[D / X B]_{A \rightarrow X, 427.0 n m-431.5 n m}^{C H_{4} \rightarrow C H}=71+/-20$

$[D / X B]_{906-911 n m}^{C H_{4} \rightarrow C I}=63+/-20$

$[D / X B]_{514.3 n m}^{\mathrm{CH}_{4} \rightarrow \mathrm{CII}}=27+/-10$

In detachment, $\mathrm{D} / \mathrm{XB}$ for $\mathrm{CH}$ is found to be increased by 2.5 +/- 1.5 times: $[D / X B]_{A \rightarrow X, 427.0 n m-431.5 n m}^{C H_{4} \rightarrow C H}=177+/-44$

The value of effective $\mathrm{D} / \mathrm{XB}$ for $\mathrm{CH}_{4}$ has been previously reported in attached divertor conditions similar to the present analysis by Poschenreider as 110 (no distinction between $\mathrm{CH}_{4}$ and $\mathrm{CD}_{4}$ is reported) [13], and by Stamp as $\sim 140+/-25$ (into a hydrogen 
plasma) [14]. The value of effective $\mathrm{D} / \mathrm{XB}$ for $\mathrm{CD}_{4}$ has been previously reported by Stamp as $\sim 80+/-25$ [14], by Monk as $~ 50$ [15] and by Huber as $\sim 80+/-20$ [16]. Data for CD4 is well summarized by Philipps [17]. Production efficiency for each monitored species above may also be derived from knowledge of the rate of molecular injection for each shot. In attached divertor conditions these are:

$\Gamma_{\mathrm{CH}} / \Gamma_{\mathrm{CH}_{4}}=0.23+/-0.10$

$\Gamma_{C^{0}} / \Gamma_{C_{4}}=0.41+/-0.15$

$\Gamma_{C^{+}} / \Gamma_{C_{4}}=0.20+/-0.08$

And in detached divertor conditions, production efficiency for $\mathrm{CH}$ is found to be much reduced:

$\Gamma_{\mathrm{CH}} / \Gamma_{\mathrm{CH}_{4}}=0.011+/-0.003$

Modelling of the PPI injection experiment to compare results using available hydrocarbon breakup reaction databases is planned with the OEDGE Monte Carlo code including DIVIMP-HC which follows transport and breakup of molecular methane [18].

As discussed above, the existence of significant uncertainty in the data presented here prevents at this time definitive conclusions regarding the contribution of chemical sputtering as a source of $\mathrm{C}^{+}$-ions entering the plasma. However, an improved version of the PPI is planned and will include a flow restrictor allowing precise flow rate determination and a built-in Langmuir probe to monitor possible local plasma perturbation caused by the gas puff. Planned experiments include operation in both attached and detached plasma conditions, with $\mathrm{CH}_{4}, \mathrm{CD}_{4}$ and heavier hydrocarbon injection.

\section{Conclusions}

The DiMES Porous Plug injector has been demonstrated to be a useful diagnostic instrument for study of plasma surface interaction phenomena in the DIII-D divertor. Data from this first use of the PPI indicate that this diagnostic has the potential of 
providing definitive information on the role of chemical sputtering, as a contributor to Catom and $\mathrm{C}^{+}$-ion sources entering the divertor plasma. From the present data it can only be concluded that chemical sputtering contributes $\sim 30-90 \%$ of the $\mathrm{C}^{+}$-source in attached divertor conditions in DIII-D. For detached conditions the contribution is much smaller. It is expected that reduction of the uncertainty bounds will be achieved with a number of improvements to the PPI, now in progress. This work was performed under the auspices of the U.S. Department of Energy by UC, LLNL under Contract W-7405-Eng-48.

\section{References}

[1] A.G. McLean, et al., Rev Sci Inst, submitted.

[2] P.C. Stangeby, et al., J Nucl Mater 313-316 (2003) 883.

[3] J. Roth, et al, J Nucl Mater 266-269 (1999) 51.

[4] Whyte, et al. J Nucl Mater 290-293 (2001) 356.

[5] N.H. Brooks, et al., Rev Sci Inst 63 (1992) 5167.

[6] N.H. Brooks, et al., PSI-17, P1-5.

[7] D. Whyte, et al., Physica Scripta T111 (2004) 34.

[8] T. Schwarz-Selinger, A. von Keudell, and W. Jacob, J Appl Phys 86 (1999) 3988.

[9] Roithova, et al., J Phys Chem B 106 (2002) 8293.

[10] A. Horn, et al., Surf Sci 331-333 (1995) 178.

[11] S. Brezinsek, et al., Physica Scripta T111 (2004) 42.

[12] E.M. Hollmann, et al., Plasma Phys Cont Fusion, submitted.

[13] W. Poschenrieder, et al., J Nucl Mater 220-222 (1995) 36.

[14] M.F. Stamp, et al., Physica Scripta T91 (2001) 13.

[15] R.D. Monk, et al., Physica Scripta T81 (1999) 54.

[16] A. Huber, et al., Physica Scripta T111 (2004) 101.

[17] V. Philipps, et al., J Nucl Mater 313-316 (2003) 354.

[18] J.D. Elder, et al., PSI-17, P1-9. 


\section{Figure captions}

Fig. 1 a) Cutaway diagram showing the design of the DiMES Porous Plug Injector. The upper portion is the DiMES sample which is topped by the porous cap. The lower section contains the gas canister, flow valve, and electrical control connections.

The porous cap is shown in b) before and, c), after plasma exposure in DIII-D.

Fig. 2 Temporal evolution of $\mathrm{CD} / \mathrm{CH}$ band structure from purely CD before onset of the PPI puff, at the puffs initiation, and later in the puff as the gas flow is increased.

Fig. 3 a) CII vs. CD/CH integrated emission counts for all PPI gas puff data. Data from 5 shots with an attached OSP and one with a detached OSP are shown. Best and bounding linear fits are shown for both cases.

b) CII vs. CD integrated intrinsic carbon source emission counts are shown. Data for each shot are averaged over the full OSP dwell. Linear fits from a) are plotted to show what fraction of CII emission can be accounted for by chemical sources. A grey box is shown to indicate bounds for calculation of the source ratio for attached divertor conditions with known errors.

\section{Figures}

a)

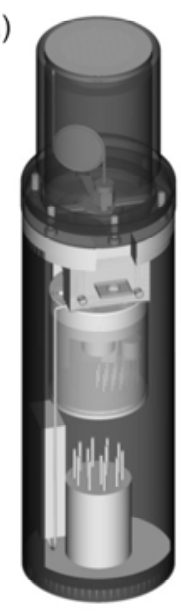

b)

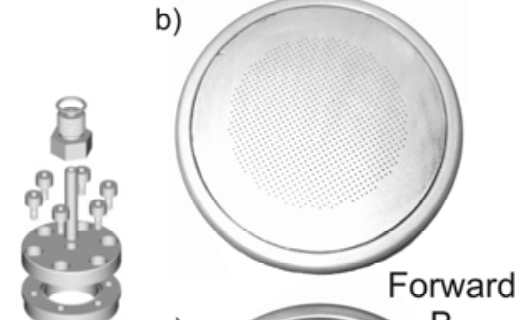

c)

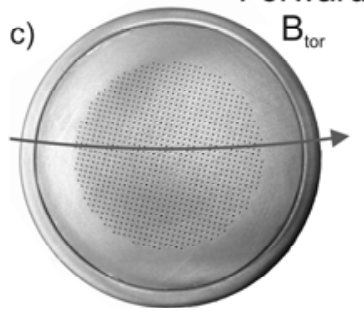

Fig. 1 


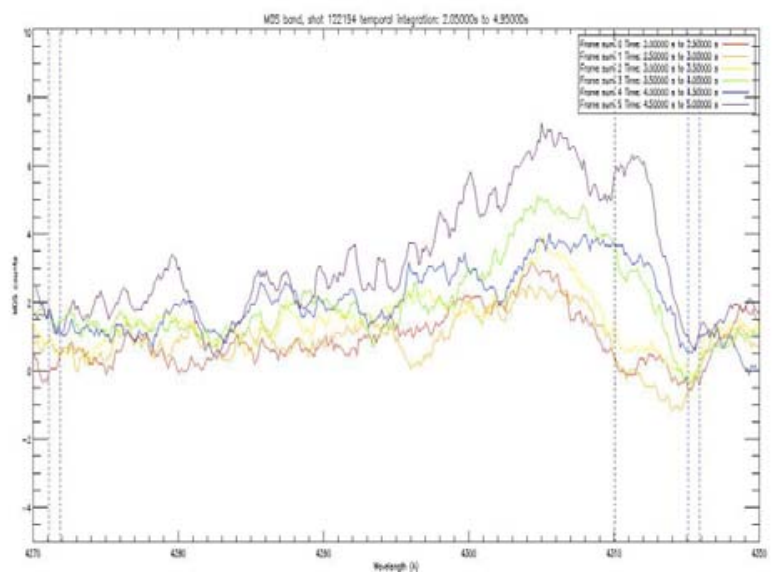

(lines need thickening up, remove legend, increase text size) Fig. 2
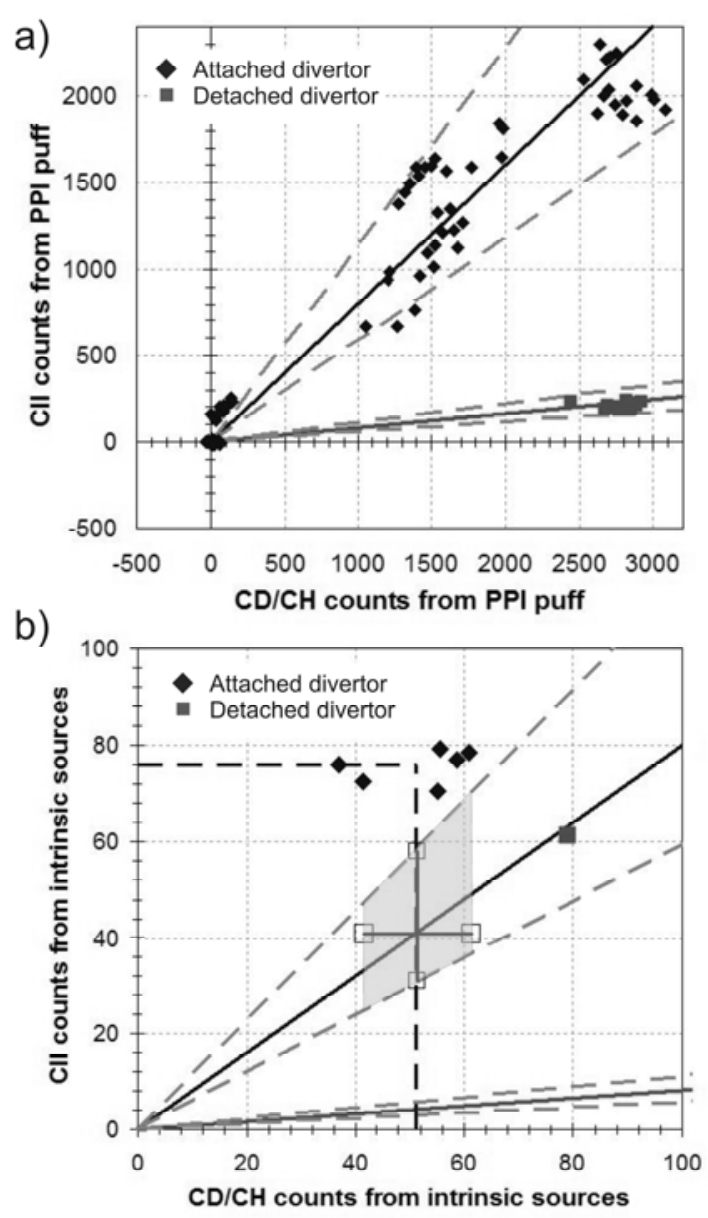

Fig. 3 